\title{
Dynamic vibration absorber for shaft machining
}

\author{
Oleg Drachev*, Igor Turbin, Irina Amirdzhanova, Tatyana Varentsova, and Veronika \\ Petrova
}

Togliatti State University, Togliatti, Russian Federation

\begin{abstract}
The article is concerned with a new method that minimizes the vibration level of non-rigid axisymmetric parts on lathes. A schematic diagram of the vibration absorber control path is given. Its configuration is changing due to fictitious elastic stiffness parameters, as well as damping and the part-support subsystem weight. A functional dual circuit diagram of an automatic vibration level control system for non-rigid shafts machining is presented and its working principle is described
\end{abstract}

Cutting process is the main source of forced vibrations in the operation of metal-cutting machines. It is possible to minimize vibration during cutting in various ways. These are relatively simple process techniques and special vibration absorbers. Vibrations can also be reduced by increasing the rigidity of the TOS (machining process system), using tools with improved geometry, optimizing cutting modes, and using dynamic vibration absorbers. The disadvantage of a dynamic vibration absorber is that it operates only at a certain frequency that corresponds to its resonant mode. Linear dynamic vibration absorbers that restore the elastic suspension forces are effective only in the case of strictly fixed excitation frequencies of rigid parts of the shaft type, and therefore their use is limited [2, 3, 4]. Even minor changes in the frequency of vibration sharply reduce the effectiveness of its operation, since it is removed from the resonant mode. Linear dynamic vibration absorbers that restore the elastic suspension forces are effective only in the case of strictly fixed excitation frequencies of rigid parts of the shaft type, and therefore their use is limited [6]. It is advisable to use such vibration absorbers in systems that have a time-constant vibration spectrum. Vibration absorbers equipped with automatic control systems are the most efficient in terms of minimizing the level of vibrations $[1,5]$. The use of nonlinear elastic elements in dynamic absorbers slightly expands the scope of their application. If the parameters of the absorber are selected properly it allows preventing the unacceptable resonant vibrations in the process of shafts machining in a wider range of excitation frequencies. However, when the excitation frequency changes over a wider range (for example, in transient modes), nonlinear dynamic vibration dampers also turn out to be ineffective, and in these cases, it is advisable to design controlled vibration absorbers with automatic frequency adjustment [5]. Changing fictitious parameters method is proposed of the part-support system using a feedback loop to ensure minimization during cutting. The schematic diagram of the system under consideration is shown in Fig. 1.

\section{*E-mail:doi05@mail.ru}




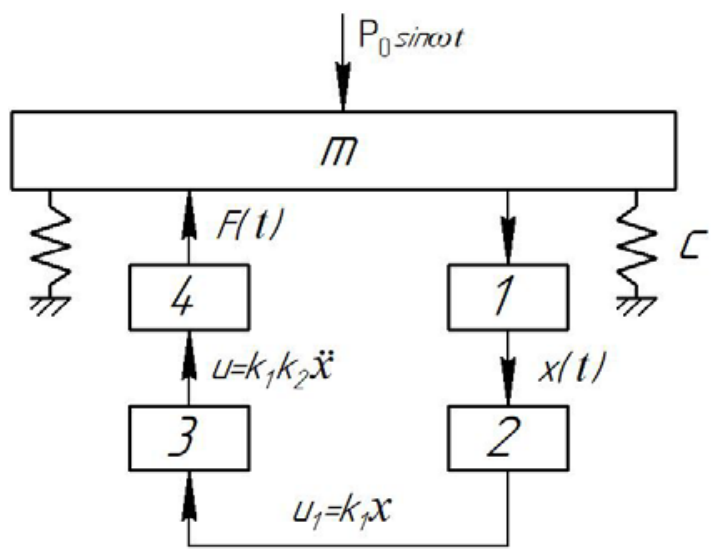

Fig. 1. Schematic diagram of the vibration absorber control path

In this case, $m$ (the weight of the shaft or axisymmetric part), which oscillates under $P_{o}$ sinwt, is supported by elastic bonds $c$ and a controlled vee 4. A displacement gage 1 is connected to $m$ and its output signal $x(t)$ is fed to the input of amplifier 2 . Then it is pre-amplified and fed to the differentiating device 3 , and then to the controlled electro-hydraulic support of the vee 4 , which is attached to a vibrating shaft that has a weight $m$.

$$
m \ddot{x}+\beta \dot{x}+c x+F(x)=P_{o} \sin \omega t,
$$

where $m$ is the weight of the system, $\beta$ is the attenuation coefficient, $c$ is the stiffness coefficient of elastic supports; $F(x)$ is the force that acts from the controlled electro-hydraulic support vee 4 on the oscillating system of the support shaft.

The diagram shows that these elements build an electromechanical feedback loop. It is possible to change the fictitious parameters of the part-support system by changing the parameters of this path. It is obvious that the equation of motion of the system under consideration (in the absence of a differentiating device 3) will be:

$$
F(x)= \pm K_{l} K_{2} K_{3} K_{4} x(t)
$$

where $K_{l}$ is the coefficient of proportionality of displacements $1 ; K_{2}$ is the gain of the inertia-free amplifier $2, K_{3}$ is the gain of the differentiating device 3 , and $K_{4}$ is the proportionality coefficient of the electrohydraulic support vee 4 . The coefficients $K_{1}, K_{2}, K_{3}, K_{4}$ are constant values. If $F(x)$ is substituted in equation (1), it will make

$$
m \ddot{x}+\beta \dot{x}+\left(c^{ \pm} c_{f}\right) x=P_{o} \sin \omega t,
$$

In this case, $c_{f}=K_{l} K_{2} K_{3} K_{4}$ is some fictitious rigidity of the system obtained using a feedback loop and equivalent to adding some real stiffness to the system $c=c_{\mathrm{f}}$. The double sign in equations (2) and (3) shows the fact that depending on the polarity of ends of sensor 1 or the differentiating amplifier 3 the direction of forces $F(x)$ may be different. By changing the activation sequence of the ends, it is always possible to change polarity and get different characteristics of systems that correspond to both positive and negative stiffness. The value of the introduced fictitious stiffness of the $c_{f}$ is adjusted by changing the modulus of the transmission coefficient of one of the links in the feedback loop (for example, an amplifier). Similarly, using the proposed method, it is possible to get an adjustable positive or negative attenuation introduced into the system. To do this, a device that differentiates the signal must 
be inserted into the feedback loop. Then the force acting from the electro-hydraulic support vee 4 to $t$ weight will be:

$$
F(x)= \pm K_{l} K_{2} K_{3} K_{4} d x / d t
$$

If the reported value is substituted for $F(x)$ into equation (1) then:

$$
m \ddot{x}+\left(\beta \pm \beta_{f}\right) \dot{x}+c x=P_{0} \sin \omega t,
$$

where $\beta_{f}=\beta_{1} \beta_{2} \beta_{3} \beta_{4}$ is the fictitious attenuation coefficient of the system. It is sufficient to implement double differentiation into the feedback loop to obtain an adjustable fictitious mass, as shown in Fig. 1. In this case, the force is:

$$
P(x)= \pm K_{l} K_{2} K_{3} K_{4} d^{2} x / d t^{2}
$$

the equation of the system's motion is:

$$
\left(m+m_{f}\right) \ddot{x}+\beta \dot{x}+c x=P_{o} \sin \omega t,
$$

where $m_{f}=K_{l} K_{2} K_{3} K_{4}$ is a fictitious weight.

If necessary, it's possible to use all three described types of feedback, including the three corresponding electro-hydraulic support vees 4 . Then, it is a following motion equation:

$$
\left(m \pm m_{f}\right) \ddot{x}+\left(\beta \pm \beta_{f}\right) \dot{x}+\left(c \pm c_{f}\right) x=P_{o} \sin \omega t
$$

Using this method, it is possible to adjust all parameters of a linear and nonlinear oscillatory system of any order, and change the resonant properties of the system within a wide range. Based on the designed model, the machine was developed for RV 106 lathe. The purpose of this machine was to increase the efficiency of vibration damping and improve the machining performance of long shafts. Machine for shafts machining contains a controlled support vee designed to interact with the part from the side opposite to the cutter. The controlled support vee is equipped with a hydraulic servo drive driven by power station. The support vee is controlled by an electromagnetic spool connected to the displacement gage sensor of the support vee relative to the part. The support vee is made of elastic elements. The machine has a feedback loop, consisting of the following: a vibration sensor, a vibration level block; the first differentiation block; the second differentiation block; the second servo drive; a controlled pressure reducer, the second input of which is connected to the hydraulic power station. Fig. 2 shows a logic diagram of an automatic vibration level control system for the machined shaft. 


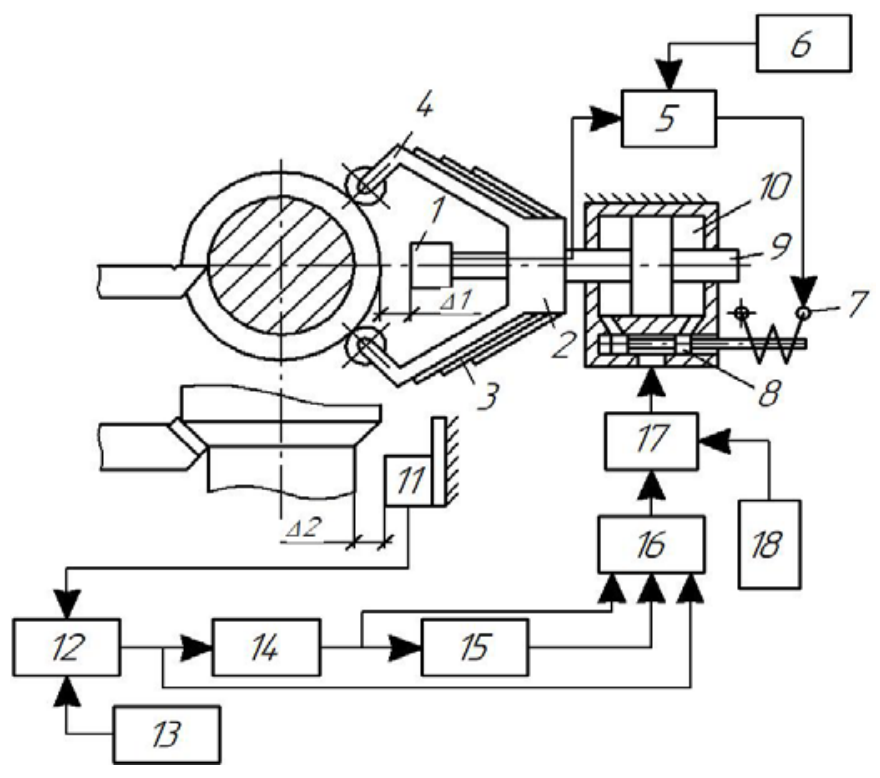

Fig. 2. Logic diagram of the automatic vibration level control system for the shaft machining

The control system consists of two paths. The first path controls the relative position of the support vee. The second path controls the parameters of the support vee servo drive. The first path includes a contactless sensor 1, rigidly fixed to the support vee 2 , which consists of elastic elements 3 and rollers 4, a differential amplifier 5, a setpoint 6 of the position of the support vee, an electromagnetic converter 7, a spool device 8, a rod-piston 9, and servo drive 10. The second path includes a level sensor 11 mounted on the machine bed, a vibration level block 12, a vibration level setter 13, a differentiation block 14, a double differentiation block 15, a servo drive 16, a controlled pressure reducer 17, and a hydraulic power station 18.

This is how the machine works. Both paths are turned on simultaneously before turning. In the first loop sensor 1, the relationship is configured with an initial gap of $\Delta_{l}$ relative to the workpiece and is mounted on a support prism 2 is made of elastic elements 3 and pressure rolls 4 , this support vee design allows to carry out force closure of parts with a support vee. Roller tension - force closure is performed by controlling the gap $\Delta$ according to the program or from the setpoint. The output signal, proportional to the vibrations of the workpiece, goes to the input of the differential amplifier 5, where it is compared with the signal of the setpoint 6 , and their difference signal is amplified and goes to the input of the electromagnetic Converter 7, where it is converted into linear movements of the spool 8, the last working pressure and controls the rod-piston 9 of the servo drive 10. The rod-piston 9 is rigidly connected to the support by a vee 2 . The second path works as follows. The vibration sensor 11 is installed with an initial gap of $\Delta$ and registers the vibration level of the workpiece relative to the machine bed. Its output signal is sent to the vibration level block 12 , where it is compared with the signal of the vibration level setter 13, and if the set vibration level is exceeded, the signal from block 12 is sent via the control circuit to the servo drive 10, which allows controlling due to the fictitious rigidity that is achieved by the feedback loop. To control the fictitious damping or dummy mass, the signal from the block 12 goes to the differentiation block 14 and then to the secondary differentiation block 15 , the output signals of the three blocks 12, 4 and 15 , each separately or sequentially switched on, then goes to the servo drive 16 and then to the controlled reducing gear 17, the pressure in which is created by the power station 18. If the parameters of the second loop are changed, proportional coefficient $K_{1}$, the sensor 11 , the coefficients $K_{2}, K_{3}$, and $K_{4}$ blocks 12,14 and 15, 
coefficients in $K_{5}$ servo drive 16 , the coefficient $K_{6}$, reducing gear 17 and the coefficient $K_{7}$ of the servo 10, and change the bogus parameters of the TOS mechanical system of the machine. Since the stiffness of the part-support subsystem will be added to the fictitious stiffness $c_{f}=K_{1}, K_{2}, K_{5}, K_{6}, K_{7}$ obtained using the feedback control loop. All elements of the control loop have linear characteristics and are inertia - free. If necessary, you can use three types of control loops, including three corresponding vibrations absorbers in terms of movement, speed, and acceleration.

The system allows changing the resonance properties of the TOS (machining process system) within wide limits as from setpoint manually or automatically by any in advance given law as the change of fictitious system parameters of TOS achieved by the control electronic elements and simultaneous change of the actual stiffness of the support vee servo drive. At the same time, the device allows for changing the fictitious parameters and non-1inear laws. It is enough to enter the non-linear element into the loop. Implementing of a controlled vibration absorber into the TOS of the RV 106 lathe makes it possible to lower the vibration level of the part-support subsystem by 10 to 12 times in the frequency range of 20 to $300 \mathrm{~Hz}$, depending on the dynamic parameters of the control system elements. The durability of the cutting tool increases by 1.5-3 times. The vibration stability of the TOS system (machining process system) of the machine increases by $6 \div 8$ times.

The vibration stability of the system during non-rigid shaft machining changes due to the movement of the movable support in the form of a self-centering collar. Therefore, to maintain vibration stability, a vibration absorber was used in conjunction with the collar.

The vibration stability of the system during non-rigid shaft machining changes due to the movement of the movable support in the form of a self-centering collar. Therefore, to maintain vibration stability, a vibration absorber was used in conjunction with the collar.

\section{References}

1. O.I. Drachev, Non-rigid axisymmetric parts manufacturing [text] / O. I. Drachev. Petersburg: Politechnika, 2005. - 290 p.: im.

2. A.V. Zharov, Determination of optimal parameters of a system with a dynamic vibration absorber. [Text] / A. V. Zharov // Siberian scientific Bulletin - Novosibirsk, 2003.-Issue 4. - P. $83-88$.

3. A.V. Zharov, Using the friction effect to increase the efficiency of vibration absorbing in systems with dynamic vibration absorbers [Text] / A. V. Zharov // Vestnik No. 6 /AltSTUafter I.Polzunova. -Barnaul, 2004. - p. 105 - 108.

4. S.Ya. Krasnovsky, V.L. Lebedev, V.I. Shapin, M Model of a controlled vibration absorber. M.: torus press publishing house, 2002.

5. Ya.G. Panovka, Introduction to the theory of mechanical vibrations. M.; Nauka 1991.-255p.

6. V.I. Zagorodsky, A.R. Maslov, Control of vibration stability of engineering systems//Komplekt ITO-2009-No. 10. P. 22-25 